\title{
Medición del valor de marca desde un enfoque formativo ${ }^{1}$
}

\section{Brand equity measurement from a formative approach}

\author{
ISABEL BuIL ${ }^{2}$ \\ Eva MARTÍNEZ \\ Leslie de Chernatony ${ }^{4}$
}

Recibido el 14 de diciembre de 2009, aceptado el 5 de marzo de 2010

$\mathrm{N}^{\circ}$ de clasificación JEL: M30

DOI: $10.5295 /$ cdg.100204ib

Reseña bibliográfica: BUIL, I.; MARTÍNEZ, E.; DE CHERNATONY, L. (2010): "Medición del valor de marca desde un enfoque formativo", Cuadernos de Gestión, Vol 10, nº especial, pp. 167-196, DOI: $10.5295 / \mathrm{cdg} .100204 \mathrm{ib}$

\section{Resumen:}

Las empresas necesitan medir el valor de sus marcas para poder tomar las mejores decisiones tácticas y estratégicas relativas a estos activos intangibles. Es por ello que este trabajo desarrolla un instrumento de medida del valor de marca utilizando un enfoque formativo. A diferencia de investigaciones anteriores, este estudio propone un modelo formativo de orden superior y valida empíricamente dicha conceptualización en dos países, España y el Reino Unido. Con este objetivo, además de realizar un análisis individual para cada país, se realiza un análisis de la invarianza en la medición siguiendo el reciente procedimiento propuesto por Diamantopoulos y Papadopoulos (2010). Los resultados obtenidos muestran que el constructo valor de marca presenta algunas diferencias en su composición entre los países analizados.

\begin{abstract}
:
Companies need to measure brand equity to make the best tactical and strategic decisions related to this intangible asset. Therefore, this paper develops a measure of brand equity using a formative approach. Unlike previous research, this study proposes a second-order formative model and empirically validates this in two countries, Spain and the United Kingdom. With this aim, in addition to carrying out an individual analysis for each
\end{abstract}

\footnotetext{
${ }^{1}$ Los autores agradecen la financiación recibida a través de los proyectos GENERÉS (Ref. S-09) del Gobierno de Aragón y el proyecto I+D+I (Ref: ECO2009-08283) de la Subdirección General de Proyectos de Investigación del Gobierno de España.

${ }^{2}$ Departamento de Dirección de Marketing e Investigación de Mercados. Escuela Universitaria de Estudios Empresariales. Universidad de Zaragoza. C/ María de Luna s/n. Edificio Lorenzo Normante. 50.018 Zaragoza. España.Email: ibuil@unizar.es

${ }^{3}$ Departamento de Dirección de Marketing e Investigación de Mercados. Facultad de Ciencias Económicas y Empresariales. Universidad de Zaragoza. Gran Vía, 2. 50005 Zaragoza. España. Email: emartine@unizar.es

${ }^{4}$ Università della Svizzera italiana, Lugano, Suiza y Aston Business School, Birmingham, Reino Unido. 50 Elizabeth Road. Moseley. Birmingham. B13 8QJ. Inglaterra. Email: dechernatony@btinternet.com
} 
country, the measurement invariance is assessed following the recent procedure suggested by Diamantopoulos and Papadopoulos (2010). Results show that brand equity construct is characterised by some variations in its composition in the countries analysed.

\section{Palabras clave:}

Valor de marca; Modelos de medida formativos; Invarianza en la medición; España; Reino Unido.

\section{Key Words:}

Brand equity; Formative measurement models; Measurement invariance; Spain; United Kingdom. 


\section{INTRODUCCIÓN}

El valor de marca desde la perspectiva del consumidor, también denominado capital de marca, es uno de los conceptos que más atención e interés ha recibido durante los últimos años en la gestión de marketing. Entendido de manera general como el valor añadido con el que la marca dota a un producto (Farquhar, 1989), la aparición de este constructo ha acentuado la importancia del papel de la marca en la organización y las estrategias de marketing durante los últimos años (Keller, 2007). Así, este concepto refleja la idea de que la marca es uno de los activos más importantes de las empresas, debido a su capacidad para proporcionar ventajas competitivas sostenibles y no imitables (Aaker, 1991; Keller, 2007).

El interés por el valor de marca ha motivado que numerosos profesionales e investigadores hayan centrado sus esfuerzos en desarrollar instrumentos de medición del mismo. En este sentido, las empresas necesitan disponer de medidas fiables y precisas que les permitan guiar el proceso de toma de decisiones y analizar la efectividad de sus estrategias (Ailawadi et al., 2003). De igual forma, los académicos precisan de este tipo de medidas para confirmar sus teorías (Arnett et al., 2009).

De entre las diferentes propuestas de medición recogidas en la literatura destacan las que describen el valor de marca como un concepto multidimensional formado por diferentes componentes medidos a su vez mediante diversos indicadores (Aaker, 1991; Keller, 1993; Yoo et al., 2000; Pappu et al., 2005; Jung y Sung, 2008; etc). La elección adecuada de estos indicadores es un aspecto muy importante en el desarrollo y validación de los instrumentos de medida. No obstante, una cuestión esencial, paralela a la selección de los indicadores más idóneos, es el examen de las relaciones epistémicas (Fornell, 1982), esto es, el vínculo existente entre el constructo y sus medidas.

En función de la dirección de este vínculo, los constructos pueden medirse a partir de dos tipos de indicadores: reflectivos y formativos (Bollen y Lennox, 1991). El uso de constructos latentes formados por indicadores reflectivos, asumiendo por tanto que la dirección de causalidad va desde el constructo hacia sus medidas, es la perspectiva dominante en la investigación en marketing (Diamantopoulos y Winklhofer, 2001). No obstante, recientemente algunos trabajos han alertado sobre los numerosos problemas que puede generar este tipo de especificación en determinadas situaciones (Diamantopoulos y Winklhofer, 2001; Edwards, 2001; MacKenzie et al., 2005; Podsakoff et al., 2006; Diamantopoulos et al., 2008). Estos trabajos proponen que en muchos casos es más recomendable el uso de indicadores (o componentes) formativos, asumiendo por tanto que los indicadores causan o forman el constructo.

En la línea de investigación del valor de marca son escasos los trabajos que han adoptado una perspectiva formativa en la conceptualización de este constructo (Delgado y $\mathrm{Mu}$ nuera, 2001; Arnett et al., 2003; Delgado, 2003). Estos estudios han utilizado en todos los casos modelos de primer orden en los que el valor de marca es medido a través de diferentes indicadores. Sin embargo, diferentes investigaciones aconsejan el uso de modelos de orden superior cuando los constructos analizados son complejos (Podsakoff et al., 2006), ya que además de que su utilización permite tratar cada dimensión como un importante componente del mismo, mejoran la representación del constructo y proporcionan el mejor modo de evaluación (MacKenzie et al., 2005). 
Asimismo, aunque algunos investigadores han realizado reiteradas llamadas de atención sobre la importancia del estudio de la marca desde un punto de vista internacional (Malhotra et al., 1999), el número de investigaciones que adoptan un enfoque internacional en la medición y análisis del proceso de creación de valor de marca es muy reducido (Yoo y Donthu, 2001; 2002; Hsieh, 2004; Erdem et al., 2006; Jung y Sung, 2008). En el caso de los constructos formativos, estos estudios cross-nacionales son si cabe todavía más escasos, ya que no ha sido hasta fechas muy recientes cuando se han ofrecido guías y procedimientos que permitan analizar la invarianza en la medición de estos constructos (Diamantopoulos y Papadopoulos, 2010).

Es por ello que este trabajo trata de avanzar sobre las investigaciones anteriores, proponiendo un modelo formativo de orden superior y validándolo en dos países. De manera más detallada, este estudio conceptualiza el valor de marca como un constructo de segundo orden con componentes formativos y valida empíricamente dicha conceptualización en un escenario cross-nacional, concretamente en dos países europeos: España y el Reino Unido. Con este fin, además de realizar un análisis individual para cada país, se realiza un análisis de la invarianza en la medición.

A través de esta conceptualización del valor de marca usando un enfoque formativo, este trabajo supera limitaciones de estudios previos, en los que se sigue el paradigma de medición dominante en marketing, esto es, el enfoque reflectivo, y avanza sobre aquellas investigaciones que, habiendo utilizado un enfoque formativo, han conceptualizado el valor de marca como un constructo de primer orden (Delgado y Munuera, 2001; Arnett et al., 2003; Delgado, 2003). Asimismo, su validación individual en cada país y el posterior análisis de invarianza en la medición permite comparar la robustez del instrumento de medición propuesto en diferentes contextos.

Para ello, el trabajo se estructura de la siguiente forma. En primer lugar se presenta una breve introducción a los modelos de medida formativos y al análisis de su invarianza. Seguidamente, se realiza una revisión de la literatura con relación a la definición y medición del valor de marca, que sirve de base para su posterior conceptualización como un constructo multidimensional de carácter formativo. A continuación se describe la metodología utilizada en el estudio y se exponen los resultados alcanzados. Finalmente, se presentan las conclusiones e implicaciones más relevantes, junto con las principales limitaciones y futuras líneas de investigación.

\section{MODELOS DE MEDIDA CON INDICADORES FORMATIVOS}

\subsection{Modelos de medida: reflectivo versus formativo}

La medición de los constructos en el ámbito del marketing requiere, de manera general, el uso de varios indicadores. Esta aproximación que asegura la validez y fiabilidad de las medidas lleva asociada de manera paralela una cuestión esencial, la modelización de la relación entre los indicadores y el constructo de interés. Tradicionalmente en la literatura se distinguen dos enfoques principales: reflectivo y formativo (Bollen y Lennox, 1991; Diamantopoulos y Winklhofer, 2001; Jarvis et al., 2003; MacKenzie et al., 2005). 
Los modelos de medida con indicadores reflectivos representan la perspectiva dominante en la investigación en marketing (Diamantopoulos y Winklhofer, 2001). Basados en la teoría de tests clásica, la principal característica de estos modelos reside en que la dirección de causalidad va desde el constructo hacia los indicadores. Por tanto, los indicadores o variables observadas constituyen un reflejo o manifestación del constructo no observado al que se encuentran ligados (Bollen y Lennox, 1991). Asimismo, en un modelo de medida de estas características los indicadores deben estar altamente correlacionados, ya que todos son un reflejo del mismo constructo, y ser intercambiables, de manera que la eliminación de alguno de ellos no debería modificar el significado de la variable objeto de estudio. Por último, en estos modelos el error está asociado a las medidas individuales.

Los modelos de medida con indicadores formativos constituyen una alternativa con gran aceptación durante los últimos años. Así, a pesar de tratarse de un enfoque cuya aparición se remonta a la década de los 60 , el interés de los investigadores por estos modelos de medida es muy reciente. Bajo este enfoque la dirección de causalidad va desde los indicadores hacia el constructo. Por tanto, los indicadores o variables observadas causan o forman el constructo no observado al que se encuentran ligados (Bollen y Lennox, 1991). Además, estos modelos de medida no requieren que los indicadores estén correlacionados. Dado que cada indicador captura un aspecto diferente del dominio conceptual de la variable, los indicadores tampoco son intercambiables. Por último, los modelos de medida con indicadores formativos incluyen un término de error que no se encuentra asociado a las medidas individuales, sino al constructo (Diamantopoulos, 2006).

Esta distinción en los modelos de medida es válida y generalizable para las estructuras factoriales de segundo orden, en cuyo caso es necesario especificar las relaciones no solo entre los indicadores y las dimensiones de primer orden, sino también entre las dimensiones de primer orden y el constructo de segundo orden (Jarvis et al., 2003; MacKenzie et al., 2005; Diamantopoulos et al., 2008).

\subsection{Procedimiento para la construcción de instrumentos de medición con indicado- res formativos}

Ante la falta de un marco metodológico que especifique las fases y requisitos necesarios para desarrollar instrumentos de medición con indicadores formativos, durante los últimos años, algunos investigadores han elaborado diferentes propuestas y guías (Diamantopoulos y Winklhofer, 2001; MacKenzie et al., 2005).

Uno de los trabajos más destacados es el de Diamantopoulos y Winklhofer (2001), en el que se proponen cuatro aspectos críticos a examinar. El primero de ellos, denominado especificación del contenido, consiste en establecer el ámbito o dominio de contenido que la variable analizada pretende capturar. Junto a éste, MacKenzie et al. (2005) incluyen además la evaluación de la dimensionalidad. Este primer aspecto es uno de los más importantes, ya que la exclusión de algunas de las facetas del constructo o una errónea especificación de la dimensionalidad del mismo supondría invalidar el instrumento de medición. El segundo aspecto, fuertemente ligado al anterior, requiere especificar los indicadores que recogen todo el significado del concepto, de acuerdo con el dominio de contenido 
anteriormente descrito. En este punto, MacKenzie et al. (2005) señalan que es necesario analizar cómo los indicadores se relacionan con el constructo o constructos de interés, distinguiendo así los indicadores reflectivos de los formativos. Una vez delimitado el contenido del concepto y el conjunto de indicadores, los dos siguientes aspectos tienen que ver con la valoración de la idoneidad del instrumento de medición con indicadores formativos. En concreto, el primero de ellos hace referencia a la colinealidad de los indicadores. Los modelos de medida formativos están basados en una regresión múltiple. Por tanto, una elevada colinealidad entre los indicadores dificultaría identificar el efecto que cada uno de ellos tiene sobre el constructo estudiado. Finalmente, el último aspecto tiene que ver con la validez externa. La naturaleza de los modelos formativos hace inadecuada su valoración atendiendo a los criterios clásicos de consistencia interna de aplicación en los indicadores reflectivos. Ante esta situación, Diamantopoulos y Winklhofer (2001) ofrecen diferentes opciones. En primer lugar destaca la estimación de un modelo MIMIC que incorpore los indicadores que componen el constructo a medir junto con varios indicadores reflectivos que resuman la esencia del concepto. Otra alternativa conlleva vincular el instrumento de medición con otros constructos con los que teóricamente se relaciona (por ejemplo, antecedentes o consecuencias).

\subsection{Evaluación de la invarianza en la medición de constructos formativos}

La valoración de la invarianza en la medición es un aspecto clave en el estudio y análisis de cualquier concepto desde un punto de vista internacional cuando se persigue realizar comparaciones entre los grupos analizados (Steenkamp y Baumgartner, 1998; Vandenberg y Lance, 2000). La falta de equivalencia puede perjudicar gravemente la validez de los resultados obtenidos (Hult et al., 2008).

De entre las diferentes técnicas propuestas en la literatura para su valoración, el análisis factorial confirmatorio multigrupo se encuentra entre las más utilizadas. Así, algunos investigadores han elaborado diferentes propuestas y paradigmas integradores que persiguen servir de ayuda en la valoración de esta cuestión a través de este enfoque. En el área de marketing destaca el trabajo de Steenkamp y Baumgartner (1998). En función del número y tipo de parámetros que permanecen invariantes entre los grupos analizados, estos autores distinguen múltiples formas de invarianza. Los tres niveles básicos de invarianza y, por ello, los que con más frecuencia aparecen en la literatura, constituyen la invarianza en el modelo o invarianza configural (configural invariance), la invarianza métrica (metric invariance) y la invarianza escalar (scalar invariance) (Steenkamp y Baumgartner, 1998). Sin embargo, tanto esta técnica como el resto de técnicas propuestas son únicamente apropiadas para los constructos reflectivos (Diamantopoulos y Papadopoulos, 2010).

No ha sido hasta fechas muy recientes cuando esta cuestión ha sido tratada para el caso de los constructos formativos (Diamantopoulos y Papadopoulos, 2010). Teniendo en cuenta las diferencias existentes entre los constructos reflectivos y formativos, Diamantopoulos y Papadopoulos (2010) proponen tres tipos de invarianza: la invarianza en la estructura (structure invariance), la invarianza en los pesos (slope invariance) y la invarianza en los residuos (residual invariance). 
La invarianza en la estructura constituye la forma más elemental de invarianza. Este tipo de invarianza implica que los indicadores que definen la estructura de la medida formativa presentan la misma configuración de pesos sustanciales y no sustanciales entre las muestras estudiadas. Su análisis no requiere la imposición de ninguna restricción, constituyendo la base para realizar las comparaciones posteriores. La invarianza en los pesos proporciona un test más riguroso. Su análisis requiere imponer la restricción de igualdad en los pesos y, por tanto, su cumplimiento implica que cada indicador influye en el mismo grado sobre el constructo formativo. Por último, la invarianza en los residuos recoge el grado en el que la varianza del término de error es la misma en los diferentes países analizados y requiere imponer la restricción de igualdad en los residuos.

Como se ha señalado anteriormente, la naturaleza de los modelos formativos hace inapropiada su valoración atendiendo a los criterios clásicos de consistencia interna. Por ello, Diamantopoulos y Papadopoulos (2010), siguiendo la propuesta comentada en el punto anterior, sugieren estimar un modelo MIMIC que incorpore los indicadores que componen el constructo a medir junto con varios indicadores reflectivos que resuman la esencia del concepto para poder valorar la invarianza en la medición. En concreto, el procedimiento propuesto, dividido en tres etapas, parte de un análisis preliminar de la invarianza en la medición de los indicadores reflectivos. A este paso inicial, le seguiría la valoración de la invarianza en la estructura mediante la estimación de un modelo MIMIC en el que tan sólo se incorporarían restricciones de igualdad entre los países en los indicadores reflectivos. Por último, la tercera etapa conllevaría la introducción de las restricciones de igualdad en los pesos, en primer lugar, y en los residuos, posteriormente, para valorar los subsiguientes niveles de invarianza.

La comprobación de la existencia de las diferentes formas de invarianza se realiza a través de una secuencia de modelos anidados jerárquicamente. La existencia de diferencias no significativas en el ajuste entre los modelos anidados indica que las restricciones impuestas pueden ser sostenidas y que, por tanto, los diferentes niveles de invarianza son satisfechos. En la evaluación del ajuste de los modelos anidados se utilizan los índices tradicionalmente recomendados en la literatura metodológica: chi-cuadrado, el error de aproximación cuadrático medio (RMSEA), el índice de ajuste comparativo robusto (CFI), el índice de ajuste incremental robusto (IFI), el índice de ajuste normado de Bentler y Bonnet (NFI) y el índice de ajuste no normado de Bentler y Bonnet (NNFI). Por último, el test de los multiplicadores de Lagrange (LM test) se utiliza para identificar los parámetros no adecuados, como por ejemplo, restricciones de igualdad que varían entre los grupos.

Al igual que en el caso de los constructos reflectivos, Diamantopoulos y Papadopoulos (2010) reconocen que conseguir estos diferentes niveles de invarianza en la práctica es complejo. Por ello, en oposición a la invarianza completa, estos autores señalan la posibilidad de alcanzar un nivel de invarianza parcial. Especialmente relevante en el caso de la evaluación de la invarianza en los pesos, este concepto reflejaría que algunos de los indicadores varían en los países objeto de estudio.

El presente trabajo toma como referencia las guías propuestas por Diamantopoulos y Winklhofer (2001) y MacKenzie et al. (2005) para la construcción del instrumento de medición del valor de marca como un constructo de segundo orden con componentes formativos. Asimismo, sigue el procedimiento recientemente propuesto por Diamantopoulos y Papadopoulos (2010) para realizar la valoración de la invarianza en la medición. En los dos 
siguientes apartados, por tanto, se especifica el dominio del constructo y se identifican las dimensiones que lo forman. Además, se justifica por qué el valor de marca se relaciona de manera formativa con sus dimensiones. Posteriormente, tras describir la metodología en la que se detallan los ítems utilizados en la medición de los diferentes componentes incluidos, se valida empíricamente dicha conceptualización. Tras comprobar la ausencia de multicolinealidad, la validación se realiza de manera individual en los dos países seleccionados: España y Reino Unido. Finalmente, se evalúa la invarianza en la medición.

\section{EL VALOR DE MARCA}

El valor de marca puede ser definido desde diferentes perspectivas y medido de múltiples formas alternativas. Cabe contraponer, no obstante, la existencia de dos enfoques fundamentales que tratan de dar respuesta a las motivaciones financieras y estratégicas que subyacen en la medición de este constructo.

$\mathrm{El}$ primer enfoque, desde una perspectiva financiera, se centra en los beneficios o resultados monetarios que la marca puede aportar a las organizaciones. Por tanto, en él se enfatiza el papel de la marca como activo de las empresas con capacidad para afectar a los flujos de caja, el valor de las acciones o el precio de venta en caso de adquisición, absorción o fusión (del Río et al., 2002). Con fines tan variados como los contables o las operaciones de fusiones o adquisiciones, las propuestas de medición bajo esta perspectiva utilizan información relativa a los costes, al valor de mercado de la empresa o al potencial de beneficios futuros para estimar el valor de marca (Winters, 1991; Seetharaman et al., 2001; Keller, 2007).

La segunda aproximación a este concepto, objeto de este estudio, parte de la premisa de que el valor de las marcas reside en la mente de los consumidores (Leone et al., 2006). En consecuencia, esta perspectiva basada en el consumidor considera a éste como la pieza clave en el proceso de medición. En esta línea se sitúan las definiciones de Keller (1993, 2007), quién define el valor de marca como el efecto diferencial que el conocimiento del nombre de marca ejerce sobre la respuesta del consumidor a las acciones de marketing, o de Aaker (1991, 1996), quién delimita el valor de marca al "conjunto de activos y pasivos vinculados a una marca, su nombre y su símbolo, que añaden o sustraen valor suministrado por un producto o servicio a la compañía y/o a sus clientes". Al contrario que en el primer enfoque, los instrumentos de medición propuestos bajo esta perspectiva se caracterizan por la utilización de distintas variables relacionadas con las percepciones, preferencias y los comportamientos de los consumidores, presentando, en la mayor parte de los casos, la ventaja de ofrecer información que las empresas pueden utilizar posteriormente en el desarrollo de sus estrategias (Christodoulides y de Chernatony, 2004).

Dentro de las medidas recogidas bajo este segundo enfoque, algunos trabajos han desarrollado criterios de medición del valor de marca unidimensionales. Entre estas propuestas destaca la utilización del precio primado (Blackston, 1990; Aaker, 1991; Swait et al., 1993; Aaker, 1996; Fernández y González, 2006) o los ingresos primados obtenidos por la marca (Ailawadi et al., 2003; Park et al., 2009). Sin embargo, la conceptualización multidimensional del valor de marca por parte de muchos autores ha favorecido la aparición de instrumentos de medición en los que se incluyen múltiples dimensiones. En este sentido, una 
revisión de la literatura pone de manifiesto que son muchas las variables consideradas por los diferentes investigadores, aunque con carácter general, Keller y Lehmann (2006) enumeran la notoriedad, las asociaciones, la actitud, la fidelidad (yendo desde la lealtad hasta la adicción) y la actividad de la marca (término referido al grado en el que los consumidores usan la marca, hablan con otros consumidores sobre la misma o buscan información, promociones o eventos, etc.) como cinco de los principales componentes.

Dentro de este grupo de métodos de medición de carácter multidimensional destacan las aportaciones realizadas por Aaker (1991, 1996) y Keller (1993), al sustentarse sobre ellas la mayor parte de los trabajos. De acuerdo con Aaker (1991) el valor de marca se conceptualiza como una entidad multidimensional formada por cinco componentes: la lealtad a la marca, la notoriedad, la calidad percibida, las asociaciones de la marca y otros activos vinculados a la marca, como las patentes. No obstante, esta última dimensión no es considerada en muchos de los estudios que siguen el enfoque de Aaker (1991), al no estar relacionada con el consumidor. Por su parte, Keller (1993) propone abordar la medición del valor de marca a través de las dimensiones que integran el conocimiento de la enseña, esto es, la notoriedad de la marca y las asociaciones unidas a la misma. En este sentido, la aproximación de medición del valor de marca supone evaluar la notoriedad, así como las características de las asociaciones de la marca -tipología, fortaleza, contenido favorable y singularidad-y las relaciones existentes entre ellas.

Tal y como se muestra en la tabla 1, en la que se sintetizan las variables más relevantes utilizadas en la literatura siguiendo este enfoque multidimensional, las dimensiones propuestas por Aaker (1991) y Keller (1993) aparecen como las más utilizadas en las investigaciones sobre el valor de marca. Por tanto, tomando como referencia estas propuestas, y a pesar de que son muchas las variables que pueden determinar el valor de una marca, este trabajo se centra en las cuatro que mayor aceptación han tenido en la literatura: la notoriedad de la marca, la calidad percibida, las asociaciones del nombre de marca y la lealtad a la marca. A continuación se comentan brevemente los aspectos más importantes de cada uno de estos componentes.

Tabla 1

Dimensiones del valor de marca identificadas en la literatura

\begin{tabular}{|c|c|}
\hline Dimensiones & Autor/es \\
\hline Notoriedad & $\begin{array}{l}\text { Aaker (1991); Keller (1993); Cobb-Walgren et al. (1995); Yoo et al. } \\
\text { (2000); Villarejo (2001); Yoo y Donthu (2001; 2002); Washburn y Plank } \\
\text { (2002); Arnett et al. (2003); Ashill y Sinha (2004); Atilgan } \text { et al. (2005); } \\
\text { Bauer } \text { et al. (2005); Kim y Kim (2005); Pappu } \text { et al. (2005; 2006; 2007); } \\
\text { Wang et al. (2006); Bravo et al. (2007); Konecnik y Gartner (2007); } \\
\text { Mohd } \text { et al. (2007); Jung y Sung (2008); Lee y Back (2008) }\end{array}$ \\
\hline Calidad percibida & $\begin{array}{l}\text { Martin y Brown (1990); Aaker (1991); Cobb-Walgren et al. (1995); Yoo et } \\
\text { al. (2000); Villarejo (2001); Yoo y Donthu (2001; 2002); Washburn y Plank } \\
\text { (2002); Arnett et al. (2003); Netemeyer et al. (2004); Atilgan et al. (2005); } \\
\text { Kim y Kim (2005); Pappu et al. (2005; 2006; 2007); Wang et al. (2006); Bra- } \\
\text { vo et al. (2007); Konecnik y Gartner (2007); Jung y Sung (2008) }\end{array}$ \\
\hline
\end{tabular}




\begin{tabular}{|c|c|}
\hline Dimensiones & Autor/es \\
\hline $\begin{array}{l}\text { Imagen de marca/ } \\
\text { asociaciones de la } \\
\text { marca }\end{array}$ & $\begin{array}{l}\text { Martin y Brown (1990); Aaker (1991); Keller (1993); Cobb-Walgren } \\
\text { et al. (1995); Yoo et al. (2000); Villarejo (2001); Yoo y Donthu (2001; } \\
\text { 2002); Washburn y Plank (2002); Vázquez et al. (2002); Arnett et al. } \\
\text { (2003); Ashill y Sinha (2004); Atilgan et al. (2005); Bauer } \text { et al. (2005); } \\
\text { Kim y Kim (2005); Pappu et al. (2005; 2006; 2007); Wang } \text { et al. (2006); } \\
\text { Koçak et al. (2007); Bravo et al. (2007); Konecnik y Gartner (2007); } \\
\text { Mohd } \text { et al. (2007); Jung y Sung (2008); Lee y Back (2008) }\end{array}$ \\
\hline Lealtad & $\begin{array}{l}\text { Aaker (1991); Yoo et al. (2000); Villarejo (2001); Yoo y Donthu (2001; } \\
\text { 2002); Washburn y Plank (2002); Arnett et al. (2003); de Chernatony et } \\
\text { al. (2004); Atilgan et al. (2005); Kim y Kim (2005); Pappu et al. (2005; } \\
\text { 2006; 2007); Wang et al. (2006); Bravo et al. (2007); Konecnik y Gartner } \\
\text { (2007); Mohd et al. (2007); Jung y Sung (2008) }\end{array}$ \\
\hline Valor percibido & Martin y Brown (1990); Netemeyer et al. (2004) \\
\hline $\begin{array}{l}\text { Compromiso y } \\
\text { confianza }\end{array}$ & Martin y Brown (1990); Lassar et al. (1995); Kim et al. (2008) \\
\hline $\begin{array}{l}\text { Rendimiento, imagen } \\
\text { social y valor }\end{array}$ & Lassar et al. (1995); Kim et al. (2008) \\
\hline Satisfacción & de Chernatony et al. (2004); Wang et al. (2006) \\
\hline Reputación & de Chernatony et al. (2004) \\
\hline Singularidad & Netemeyer et al. (2004); Mohd et al. (2007) \\
\hline
\end{tabular}

Fuente: Elaboración propia.

La notoriedad de la marca recoge la capacidad potencial que un consumidor tiene para reconocer o recordar el nombre de una marca como miembro de una cierta categoría de producto (Aaker, 1991). Sobre la base del modelo de memoria de red asociativa, Keller (1993) señala que esta dimensión está relacionada con la fortaleza de la marca en la memoria de los consumidores, reflejándose dicha fortaleza en la capacidad del consumidor para identificar la marca bajo diferentes condiciones (Rossiter y Percy, 1987). La notoriedad constituye una de las condiciones necesarias para que una marca posea valor, ya que es imprescindible que el consumidor pueda identificar la enseña en diferentes situaciones. No obstante, representa el nivel más bajo de conocimiento posible sobre la marca (Delgado, 2003).

La calidad percibida se define como "la percepción que el cliente tiene sobre la calidad general o superioridad de un producto o servicio respecto a su propósito entre alternativas" (Zeithaml, 1988). Se trata, por tanto, de una valoración subjetiva de la calidad del producto o servicio. Esta dimensión se menciona comúnmente en la literatura como uno de los componentes clave en la construcción de una marca fuerte, al ser la base para una evaluación favorable y positiva por parte de los consumidores, desde un punto de vista cognitivo, afectivo y de comportamiento (Farquhar, 1989). Además se relaciona con la mayor diferenciación y superioridad de la enseña, la disposición del consumidor a pagar un precio primado y la elección e intención de compra (Netemeyer et al., 2004). 
La dimensión de asociaciones de la marca es otro de los componentes clave del valor de marca (Aaker, 1996; Chen, 2001). Concretamente, Aaker (1991) define las asociaciones de la marca como "cualquier cosa unida en la memoria a una marca". De modo similar, Keller (1993) afirma que las asociaciones están unidas al nodo de la marca en la memoria y contienen el significado de la marca para los consumidores. Estas asociaciones de la marca pueden consistir en descripciones verbales, visuales, impresiones sensoriales (un sabor, aroma o sonido) y emocionales (Supphellen, 2000), que finalmente dan lugar a una red sólida de conocimientos sobre la marca organizadas en la memoria del consumidor. Este componente tiene gran relevancia en la gestión del valor de marca (Aaker, 1991; Chen, 2001) al proporcionar numerosas ventajas competitivas (del Río et al., 2001). Así, a través de las asociaciones, las organizaciones pueden diferenciar y posicionar sus productos, y crear actitudes y sentimientos positivos hacia su marca (Aaker, 1991; Low y Lamb, 2000; Dean, 2004), lo cual puede traducirse en una mayor predisposición a adquirir o usar el producto (Yoo et al., 2000; Chen, 2001).

Finalmente, la lealtad es uno de los componentes del valor de marca que más interés y estudio ha recibido en la literatura de marketing. Definida como la medida del vínculo existente entre un cliente y la marca (Aaker, 1991), esta dimensión representa para muchos estudios el pilar esencial del valor de una enseña (Yoo et al., 2000; Atilgan et al., 2005; Mohd et al., 2007). La lealtad es un activo estratégico que, adecuadamente gestionado, puede generar importantes ventajas para las organizaciones. En este sentido, los consumidores leales muestran respuestas más favorables hacia la marca que los no leales (Grover y Srinivasan, 1992). Por tanto, muchos trabajos tienen en cuenta esta dimensión en la conceptualización del valor de marca.

\section{CONCEPTUALIZACIÓN DEL VALOR DE MARCA: UN MODELO FORMA- TIVO DE ORDEN SUPERIOR}

El valor de marca, a la vista de lo expuesto en el apartado anterior, se conceptualiza como un constructo multidimensional, dando lugar a un modelo de orden superior. Tal y como Podsakoff et al. (2006) señalan, el uso de modelos de orden superior es recomendable cuando los constructos analizados son complejos, ya que su utilización permite tratar cada dimensión como un importante componente del mismo. Además, estos modelos mejoran la representación del constructo y proporcionan el mejor modo de evaluación (MacKenzie et al., 2005). En consecuencia, la conceptualización realizada resulta apropiada. El siguiente paso consiste, por tanto, en determinar si estas dimensiones se relacionan con el constructo de segundo orden de manera reflectiva o formativa.

Siguiendo los criterios propuestos por Jarvis et al. (2003) y MacKenzie et al. (2005), relativos a la dirección de causalidad entre el constructo y sus componentes, la intercambiabilidad de las dimensiones, la covarianza de las medidas y la similitud de las redes nomológicas de los componentes, este trabajo considera que el valor de marca está relacionado de manera formativa con sus dimensiones. Así, en primer lugar, se propone que no es el valor de marca quien causa sus dimensiones, sino al contrario. Esto es, cambios en los componentes del valor de marca, como las asociaciones o la lealtad, causan cambios en el valor de la enseña. Además, las dimensiones no son intercambiables, al capturar diferentes 
aspectos del dominio conceptual de la variable estudiada. Con relación al tercer criterio, relativo a la covarianza de las medidas, no hay por qué esperar que todas las dimensiones presenten fuertes correlaciones. Por ejemplo, un cambio en la notoriedad de la marca no tiene por qué estar acompañado de un cambio en la calidad percibida de la misma. Por último, los componentes integrantes del constructo valor de marca no comparten en su totalidad los mismos antecedentes y consecuencias.

En la línea de investigación del valor de marca, un número reducido de trabajos ha adoptado una perspectiva formativa en la conceptualización y operacionalización de este constructo (Delgado y Munuera, 2001; Arnett et al., 2003; Delgado, 2003). En concreto, estas investigaciones miden el valor de marca a partir de modelos formativos de primer orden.

Por tanto, a diferencia de los estudios previos existentes, este trabajo conceptualiza el valor de marca a partir de una estructura de orden superior. De este modo, como se ilustra en la figura 1, el constructo valor de marca queda especificado como una variable agregada de segundo orden con cuatro constructos latentes de primer orden formativos. Por último, cada uno de estos constructos latentes de primer orden es medido a través de múltiples indicadores reflectivos.

Figura 1

Modelo de medida del valor de marca

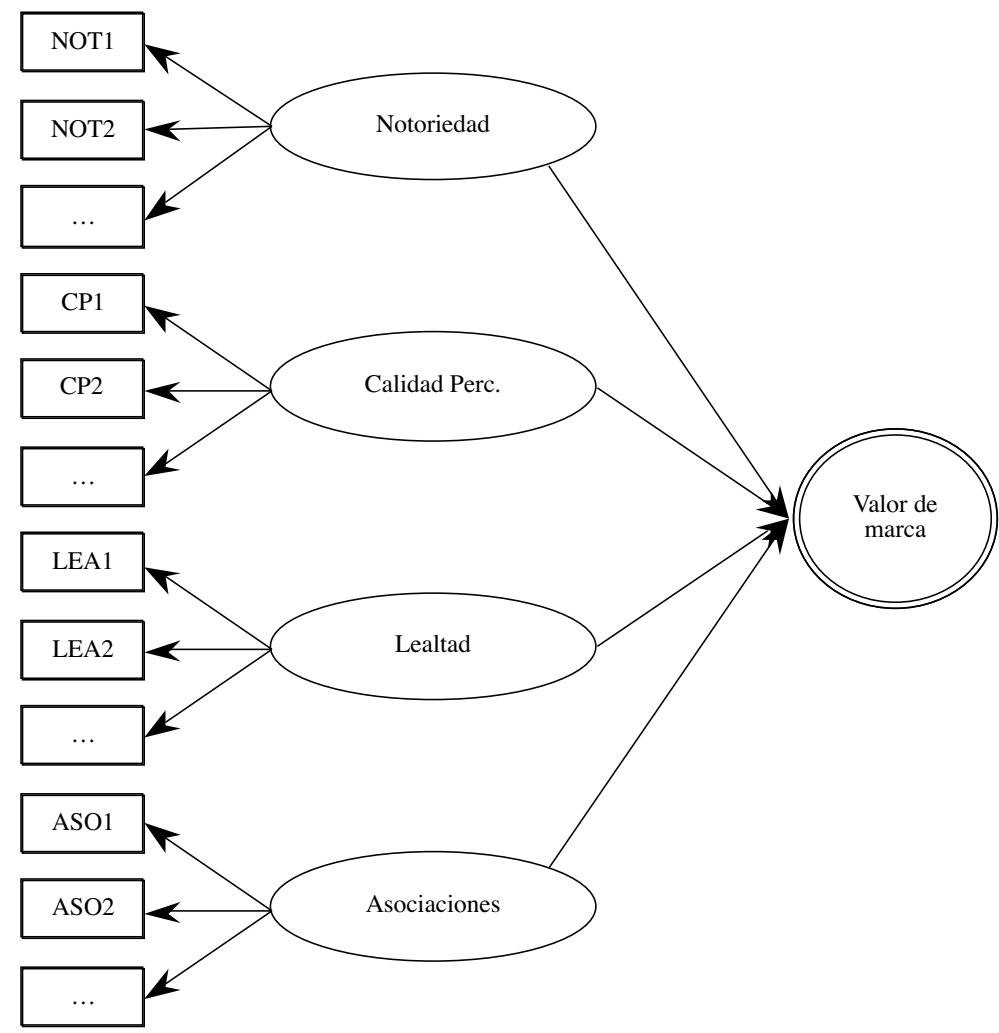




\section{METODOLOGÍA}

Para validar empíricamente la conceptualización formativa del constructo valor de marca se realizó un estudio de mercado en dos países: España y el Reino Unido. Esta validación en un escenario cross-nacional resulta especialmente interesante al permitir comparar la robustez de la medida en diferentes contextos.

La selección de los países obedeció a las siguientes razones. En primer lugar, se consideró interesante realizar un estudio con países europeos, ya que los escasos estudios que hasta el momento han profundizado en el análisis del valor de marca de manera simultánea en diferentes países se han centrado principalmente en comparar los EEUU con países asiáticos como Corea del Sur (Yoo y Donthu, 2002; Jung y Sung, 2008). En segundo lugar, se seleccionaron dos países con un desarrollo económico y social similar, difiriendo básicamente en sus características culturales (Kumar, 2000; Hofstede, 2001; Hsieh, 2002), con el objetivo de aumentar la generalización de los resultados.

A continuación se describe el proceso de selección de las categorías de producto y marcas, así como el de obtención de la información y selección de las muestras. Posteriormente, se presentan las escalas de medición de las variables consideradas.

\subsection{Selección de las categorías de producto y marcas}

El presente trabajo utilizó ocho marcas pertenecientes a cuatro categorías de producto para el estudio del valor de marca. En la selección de las categorías de productos y marcas se valoró su función principal y su conocimiento y disponibilidad entre los consumidores de ambos países. Además, para ampliar el alcance y la generalización de los resultados, se consideró interesante la selección de categorías de producto y marcas pertenecientes tanto a los sectores de bienes de consumo frecuente como de consumo duradero que presentasen diferencias en aspectos tales como el precio, las estrategias de marketing mix, la frecuencia de compra, etc.

El uso de rankings e informes externos en la selección de los productos y marcas es una práctica habitual en las investigaciones sobre el valor de marca (Cobb-Walgren et al., 1995; Krishnan, 1996; Yoo et al., 2000; Yoo y Donthu, 2001; Netemeyer et al., 2004). De este modo, este estudio tomó como referencia el ranking de Interbrand "Las mejores marcas globales 2006". Tras un análisis del mismo, atendiendo a las necesidades de la investigación anteriormente apuntadas, se optó por elegir las siguientes categorías de producto: refrescos, ropa y calzado deportivo, productos electrónicos y automóviles.

Posteriormente, para cada categoría de producto se eligieron dos marcas con una posición diferenciada dentro del ranking, con el objetivo de aumentar la variabilidad. Las marcas elegidas fueron: Coca Cola y Pepsi dentro de la categoría de refrescos; Nike y Adidas en la de ropa y calzado deportivo; Sony y Panasonic en la de productos electrónicos; y, por último, BMW y Volkswagen en la categoría de automóviles.

\subsection{Procedimiento y muestras}

La recogida de información se realizó en dos ciudades, Zaragoza (España) y Birmingham (Reino Unido), mediante encuesta personal dirigida a individuos en edades com- 
prendidas entre los 15 y 59 años. Ambas ciudades presentan semejanzas en cuanto a sus características poblacionales y son representativas de ambos mercados, lo que favorece la comparabilidad de las mismas. En total se elaboraron ocho cuestionarios, uno para cada marca, con la misma estructura y preguntas. Es importante señalar que cada encuestado respondió a una única encuesta.

El trabajo de campo fue realizado por dos grupos de encuestadores, situados en distintas zonas y momentos del día en las dos ciudades consideradas, durante los meses de febrero y abril de 2007. La selección de la muestra se realizó a partir de un muestreo por cuotas, en base a la edad y el sexo de los individuos ${ }^{5}$, al ser éste uno de los procedimientos de muestreo no probabilísticos más utilizados en las investigaciones de carácter internacional por garantizar la comparabilidad de los datos (Craig y Douglas, 2005). Como resultado de este trabajo de campo se obtuvieron un total de 417 encuestas en el Reino Unido y de 414 en España. Una vez realizado el proceso de depuración se dispuso de un total de 411 encuestas válidas en cada país. En concreto, del total de la muestra española, un 49,4\% son hombres. Asimismo, un 24,8\% de los entrevistados pertenece al segmento de edad entre 15 y 24 años, un $37,2 \%$ se sitúa entre los 25 y 39 años, mientras que el resto, un $38 \%$, lo hace entre los 40 y 59 años. En lo que respecta a la muestra inglesa, un 50,9\% son hombres, y en lo referente a la edad, un $24,3 \%$ se sitúa en el segmento entre 15 y 24 años, un 37,5\% entre 25 y 39 años, y el resto entre 40 y 59 años. Por su parte, el número de cuestionarios conseguidos para cada una de las ocho marcas es muy parecido, tanto en España como en el Reino Unido, situándose en torno a las 50 encuestas.

\subsection{Medición de las variables}

Para la medición de los diferentes componentes del valor de marca incluidos en el estudio se utilizaron escalas extraídas de investigaciones previas. La notoriedad de marca se midió en base a la propuesta de Yoo et al. (2000) y Netemeyer et al. (2004). La escala empleada para la medición de la calidad percibida, que valora este constructo de una manera global sin centrarse en los atributos específicos de una categoría concreta, se desarrolló a partir de los ítems utilizados por Pappu et al. (2005, 2006). La lealtad a la marca se midió a través de una escala adaptada de Yoo et al. (2000). Finalmente, las asociaciones de marca, se midieron a través de varios ítems propuestos por diferentes investigadores (Lassar et al., 1995; Aaker, 1996; Netemeyer et al., 2004; Pappu et al., 2005). De acuerdo con la clasificación de Aaker (1996) se consideraron tres tipos de asociaciones: valor percibido, personalidad y asociaciones de carácter organizacional.

El análisis de la validez externa de una escala formativa requiere, como se ha señalado anteriormente, la incorporación de una medida global del constructo objeto de análisis y de uno o varios constructos con los cuales se relaciona (Diamantopoulos y Winklhofer, 2001; MacKenzie et al., 2005). En consecuencia, el valor de marca también se midió utilizando una escala reflectiva propuesta por Yoo et al. (2000), generalmente utilizada para medir este constructo de manera global (Delgado y Munuera, 2001; Arnett et al., 2003). Adicionalmen-

\footnotetext{
${ }^{5}$ La fijación de las cuotas se realizó a partir del análisis de la distribución de la población en las dos ciudades seleccionadas, Zaragoza y Birmingham, con relación a las variables sexo y edad.
} 
te, la disposición del consumidor a pagar un precio primado y la intención de compra, como constructos relacionados con el valor de marca, se midieron a partir de los ítems procedentes de los trabajos de Netemeyer et al. (2004) y Erdem et al. (2006) respectivamente.

En el apéndice se recogen las escalas utilizadas. En todos los casos se utilizaron escalas tipo Likert de siete puntos ( $1=$ totalmente en desacuerdo; $7=$ totalmente de acuerdo $)$.

\section{ANÁLISIS DE RESULTADOS}

Para validar el instrumento de medición del valor de marca se recurrió a la metodología de ecuaciones estructurales utilizando el programa informático EQS 6.1. El procedimiento de validación se estructuró en dos fases. En una primera fase, dado que el valor de marca quedó especificado como un modelo reflectivo de primer orden y formativo de segundo orden, se analizaron las propiedades psicométricas de las variables incluidas medidas con indicadores reflectivos a través de diversos análisis exploratorios y confirmatorios de manera individual en cada país. Posteriormente, se examinó la multicolinealidad entre los componentes formativos y se procedió a validar el instrumento de medida propuesto en cada uno de los dos contextos estudiados atendiendo a las recomendaciones de Diamantopoulos y Winklhofer (2001) anteriormente comentadas. En una segunda fase, se llevó a cabo el examen de la invarianza en la medición como requisito previo imprescindible para realizar comparaciones entre las diferentes poblaciones estudiadas.

\subsection{Análisis individual para cada país}

\section{A. Validación de los constructos de primer orden con indicadores reflectivos}

El examen de las propiedades psicométricas de los constructos con indicadores reflectivos, esto es, los cuatro componentes del valor de marca así como los constructos adicionales incluidos (valor de marca medido con indicadores reflectivos, precio primado e intención de compra), comenzó con una serie de análisis iniciales de fiabilidad a partir del estadístico alpha de Cronbach y la correlación ítem-total, presentando en ambos países unos resultados aceptables.

Posteriormente, con el objetivo de examinar la dimensionalidad de cada escala, se realizaron diversos análisis factoriales exploratorios, utilizando como método de extracción el de componentes principales con rotación Varimax en ambas muestras. Estos análisis, justificados por los valores relativos al KMO y la prueba de esfericidad de Bartlett, mostraron que detrás de cada escala, con la excepción del componente relativo a las asociaciones de marca, subyacía un único factor. En particular, bajo el componente asociaciones de marca aparecieron tres factores que hacen referencia al valor percibido (ítems 1, 2 y 3), a la personalidad de la marca (ítems 4,5 y 6) y a las asociaciones de carácter organizacional (ítems 7, 8 y 9). Un examen en detalle de la dimensionalidad de este componente a través de análisis factoriales confirmatorios arrojó de nuevo los mismos resultados. En consecuencia, siguiendo las recomendaciones de estudiar cada dimensión integrante del constructo asociaciones de forma individual para, de este modo, guiar de una manera más efectiva las 
decisiones relativas a las mismas (del Río et al., 2001), en los análisis posteriores se incluyeron las tres dimensiones de forma independiente.

Finalmente, se realizaron análisis factoriales confirmatorios en las dos muestras con la finalidad de corroborar la fiabilidad y dimensionalidad de las escalas y demostrar su validez estadística. El primer análisis puso de manifiesto la necesidad de eliminar uno de los ítems de la escala propuesta para la medición de la personalidad de la marca (ASO6) y otro de la escala que mide la disposición a pagar un precio primado (PRE1), ya que el $\mathrm{R}^{2}$ presentó en ambos casos un valor inferior al mínimo exigido tanto en España como en el Reino Unido. Una vez eliminados estos indicadores, los resultados mostraron una adecuada especificación de la estructura factorial propuesta (tabla 2). En este sentido todos los indicadores presentaron coeficientes lambda estandarizados significativos y superiores a 0,50, lo cual constata la validez convergente de las escalas (Steemkamp y Van Trijp, 1991). Asimismo, los coeficientes presentaron una clara relación con cada uno de los factores subyacentes $\left(\mathrm{R}^{2}>0,3\right)$. En lo que respecta a los análisis relativos a la fiabilidad definitiva, los coeficientes de fiabilidad compuesta (FC) y el análisis de la varianza extraída (AVE) superaron los valores aconsejados de 0,7 y 0,5 , respectivamente (Hair et al., 2006). Con relación a la validez discriminante de los modelos de medida, ésta también fue respaldada, al comprobarse que ninguno de los intervalos de confianza de las correlaciones estimadas entre cada par de dimensiones contenía el valor 1.

Por último los indicadores de la calidad de la bondad de ajuste fueron, en general, positivos y superaron los umbrales mínimos establecidos en ambos países (Kline, 2005; Hair et al., 2006).

Tabla 2

\section{Resultados del análisis factorial confirmatorio}

\begin{tabular}{|c|c|c|c|c|c|c|c|c|c|}
\hline \multirow{2}{*}{ Variables latentes } & \multirow{2}{*}{ Ítems } & \multicolumn{4}{|c|}{ España } & \multicolumn{4}{|c|}{ Reino Unido } \\
\hline & & $\lambda(\mathrm{t})$ & $\mathrm{R}^{2}$ & FC & AVE & $\lambda(\mathrm{t})$ & $\mathrm{R}^{2}$ & FC & AVE \\
\hline \multirow{5}{*}{ Notoriedad } & NOT1. & 0,776 & 0,602 & \multirow{5}{*}{0,950} & \multirow{5}{*}{0,825} & 0,783 & 0,613 & \multirow{5}{*}{0,899} & \multirow{5}{*}{0,642} \\
\hline & NOT2. & 0,740 & 0,548 & & & 0,735 & 0,540 & & \\
\hline & NOT3. & 0,856 & 0,732 & & & 0,770 & 0,592 & & \\
\hline & NOT4. & 0,858 & 0,736 & & & 0,854 & 0,730 & & \\
\hline & NOT5. & 0,664 & 0,441 & & & 0,857 & 0,735 & & \\
\hline \multirow{4}{*}{ Calidad percibida } & CP1. & 0,909 & 0,825 & \multirow{4}{*}{0,887} & \multirow{4}{*}{0,612} & 0,885 & 0,784 & \multirow{4}{*}{0,929} & \multirow{4}{*}{0,765} \\
\hline & $\mathrm{CP} 2$. & 0,938 & 0,881 & & & 0,883 & 0,780 & & \\
\hline & $\mathrm{CP} 3$. & 0,886 & 0,785 & & & 0,928 & 0,861 & & \\
\hline & CP4. & 0,899 & 0,809 & & & 0,798 & 0,636 & & \\
\hline \multirow{3}{*}{ Lealtad } & LEA1. & 0,867 & 0,753 & \multirow{3}{*}{0,917} & \multirow{3}{*}{0,788} & 0,897 & 0,804 & \multirow{3}{*}{0,907} & \multirow{3}{*}{0,764} \\
\hline & LEA2. & 0,940 & 0,884 & & & 0,903 & 0,815 & & \\
\hline & LEA3. & 0,853 & 0,727 & & & 0,820 & 0,672 & & \\
\hline \multirow{3}{*}{$\begin{array}{l}\text { Valor percibido } \\
\text { (asoc.) }\end{array}$} & ASO1. & 0,804 & 0,647 & \multirow{3}{*}{0,873} & \multirow{3}{*}{0,697} & 0,808 & 0,653 & \multirow{3}{*}{0,943} & \multirow{3}{*}{0,806} \\
\hline & $\mathrm{ASO} 2$. & 0,897 & 0,805 & & & 0,933 & 0,871 & & \\
\hline & ASO3. & 0,800 & 0,640 & & & 0,773 & 0,598 & & \\
\hline
\end{tabular}




\begin{tabular}{|c|c|c|c|c|c|c|c|c|c|}
\hline \multirow{2}{*}{ Variables latentes } & \multirow{2}{*}{ Ítems } & \multicolumn{4}{|c|}{ España } & \multicolumn{4}{|c|}{ Reino Unido } \\
\hline & & $\lambda(\mathrm{t})$ & $\mathrm{R}^{2}$ & FC & AVE & $\lambda(\mathrm{t})$ & $\mathrm{R}^{2}$ & $\mathrm{FC}$ & AVE \\
\hline \multirow{2}{*}{$\begin{array}{l}\text { Personalidad } \\
\text { marca (asoc.) }\end{array}$} & ASO4. & 0,863 & 0,745 & \multirow{2}{*}{0,882} & \multirow{2}{*}{0,789} & 0,837 & 0,701 & \multirow{2}{*}{0,878} & \multirow{2}{*}{0,707} \\
\hline & ASO5. & 0,913 & 0,833 & & & 0,915 & 0,837 & & \\
\hline \multirow{3}{*}{$\begin{array}{l}\text { Asoc. } \\
\text { Organizacionales } \\
\text { (asoc.) }\end{array}$} & ASO7. & 0,939 & 0,881 & \multirow{3}{*}{0,953} & \multirow{3}{*}{0,781} & 0,905 & 0,819 & \multirow{3}{*}{0,869} & \multirow{3}{*}{0,769} \\
\hline & ASO8. & 0,932 & 0,868 & & & 0,892 & 0,796 & & \\
\hline & ASO9. & 0,929 & 0,863 & & & 0,885 & 0,783 & & \\
\hline \multirow{4}{*}{$\begin{array}{l}\text { Valor de marca } \\
\text { (reflectivo) }\end{array}$} & VM1. & 0,824 & 0,680 & \multirow{4}{*}{0,941} & \multirow{4}{*}{0,808} & 0,843 & 0,711 & \multirow{4}{*}{0,923} & \multirow{4}{*}{0,799} \\
\hline & VM2. & 0,944 & 0,891 & & & 0,947 & 0,897 & & \\
\hline & VM3. & 0,958 & 0,918 & & & 0,931 & 0,867 & & \\
\hline & VM4. & 0,844 & 0,712 & & & 0,867 & 0,751 & & \\
\hline \multirow{2}{*}{ Precio primado } & PRE2. & 0,946 & 0,895 & \multirow{2}{*}{0,916} & \multirow{2}{*}{0,844} & 0,959 & 0,920 & \multirow{2}{*}{0,920} & \multirow{2}{*}{0,852} \\
\hline & PRE3. & 0,891 & 0,794 & & & 0,886 & 0,785 & & \\
\hline \multirow{3}{*}{$\begin{array}{l}\text { Intención de } \\
\text { compra }\end{array}$} & COM1. & 0,878 & 0,771 & \multirow{3}{*}{0,938} & \multirow{3}{*}{0,835} & 0,918 & 0,842 & \multirow{3}{*}{0,941} & \multirow{3}{*}{0,842} \\
\hline & COM2. & 0,929 & 0,862 & & & 0,910 & 0,828 & & \\
\hline & COM3. & 0,933 & 0,870 & & & 0,924 & 0,853 & & \\
\hline \multicolumn{2}{|l|}{ Bondad de ajuste } & \multicolumn{4}{|c|}{$\begin{array}{l}\mathbf{S}-\mathbf{B} \boldsymbol{\chi}^{2}(\mathbf{d f}, \mathbf{p})=836,94(341,0,00) \\
\mathbf{N F I}=0,892 \quad \mathbf{N N F I}=0,920 \\
\mathbf{C F I}=0,932 \quad \mathbf{I F I}=0,933 \\
\mathbf{R M S E A}=0,060\end{array}$} & \multicolumn{4}{|c|}{$\begin{array}{l}\mathbf{S}-\mathbf{B} \boldsymbol{\chi}^{\mathbf{2}}(\mathbf{d f}, \mathbf{p})=700,90(341,0,00) \\
\mathbf{N F I}=0,925 \mathbf{N N F I}=0,952 \\
\mathbf{C F I}=0,960 \mathbf{I F I}=0,960 \\
\mathbf{R M S E A}=0,051\end{array}$} \\
\hline
\end{tabular}

\section{B. Validación del instrumento de medición del valor de marca}

Evaluadas las propiedades psicométricas de los constructos medidos a través de indicadores reflectivos se procedió a dar cumplimiento a los análisis de colinealidad. En concreto, el examen de la misma se realizó a través de un análisis de regresión lineal en cada país en el que se tomaron los valores medios de cada uno de los componentes como variables independientes y la media del valor de marca medida a través de los indicadores reflectivos como variable dependiente. La no colinealidad se reflejó en el factor de inflación de la varianza (FIV) con valores inferiores a 5 (tabla 3) (Belsley, 1991).

Tabla 3

Test de colinealidad

\begin{tabular}{l|c|c}
\hline \multirow{2}{*}{ Componentes } & \multicolumn{2}{c}{ Factor de inflación de la varianza (FIV) } \\
\cline { 2 - 3 } & España & Reino Unido \\
\hline Notoriedad & 1,746 & 1,433 \\
\hline Calidad percibida & 2,254 & 1,927 \\
\hline Lealtad & 1,535 & 1,632 \\
\hline Valor percibido (asoc.) & 3,122 & 2,153 \\
\hline Personalidad de la marca (asoc.) & 2,117 & 1,815 \\
\hline Asoc. organizacionales (asoc.) & 2,306 & 2,022 \\
\hline
\end{tabular}


El siguiente paso consistió en estudiar la validez externa de la escala formativa en los dos contextos analizados. Para ello, se estimaron dos modelos MIMIC que incorporaron como indicadores formativos los componentes ya comentados y como causas los indicadores reflectivos propuestos por Yoo et al. (2000) que resumen la esencia del concepto. La tabla 4 muestra los resultados obtenidos al estimar este modelo en ambos países.

Como puede observarse tres de los seis componentes considerados contribuyen a la formación del valor de marca de manera significativa en ambos países. Éstos son la lealtad y dos de las asociaciones de marca incluidas: el valor percibido y la personalidad de marca. Por su parte, la calidad percibida no ejerce una influencia significativa en ninguna de las dos muestras analizadas. Las principales diferencias radican en la notoriedad y las asociaciones organizacionales. La primera no tiene una influencia significativa en la muestra inglesa mientras que, en sentido contrario al esperado, presenta un efecto negativo y significativo en España. Por otro lado, las asociaciones organizacionales sólo influyen de manera significativa en el Reino Unido. A continuación se comentan estos resultados de manera más detallada.

Tabla 4

Resultados del modelo MIMIC

\begin{tabular}{l|c|c}
\hline \multirow{2}{*}{ Relación } & \multicolumn{2}{c}{ Parámetro estandarizado (error) } \\
\cline { 2 - 3 } & España & Reino Unido \\
\hline Notoriedad $\rightarrow$ Índice valor de marca & $-0,201 *(-3,393)$ & $-0,051(-1,023)$ \\
\hline Calidad percibida $\rightarrow$ Índice valor de marca & $0,091(1,418)$ & $0,037(0,649)$ \\
\hline Lealtad $\rightarrow$ Índice valor de marca & $0,572^{*}(7,902)$ & $0,406^{*}(6,563)$ \\
\hline Valor percibido (asoc.) $\rightarrow$ Índice valor de marca & $0,272^{*}(2,528)$ & $0,203 *(3,053)$ \\
\hline Personalidad de la marca (asoc.) $\rightarrow$ Índice valor de marca & $0,195 *(2,690)$ & $0,122^{*}(1,978)$ \\
\hline Asoc. Organizacionales (asoc.) $\rightarrow$ Índice valor de marca & $-0,070(-1,117)$ & $0,231 *(3,189)$ \\
\hline
\end{tabular}

Bondad de ajuste del modelo: España

$\mathrm{S}-\mathrm{B} \chi^{2}(\mathrm{df}, \mathrm{p})=586,93(231,0,00) ; \mathrm{RMSEA}=0,061 ;$

$\mathrm{NFI}=0,894 ; \mathrm{NNFI}=0,919 ; \mathrm{CFI}=0,932 ; \mathrm{IFI}=0,933$

\section{Bondad de ajuste del modelo: Reino Unido}

$\mathrm{S}-\mathrm{B} \chi^{2}(\mathrm{df}, \mathrm{p})=533,77(231,0,00) ; \mathrm{RMSEA}=0,057$;

$\mathrm{NFI}=0,923 ; \mathrm{NNFI}=0,945 ; \mathrm{CFI}=0,954 ; \mathrm{IFI}=0,955$

Nota: $* \mathrm{p}<0,05$

La estimación del modelo MIMIC sugiere que el valor de marca viene determinado de un modo significativo por la lealtad, el valor percibido y la personalidad de marca en ambos países. Tal y como se recoge en la tabla 4 , por orden de importancia de los pesos de 
cada uno de los componentes, en primer lugar se sitúa la lealtad $(0,572$ y 0,406 en España y el Reino Unido, respectivamente), el valor percibido $(0,272$ y 0,203$)$ y la personalidad de marca $(0,195$ y 0,122$)$. Por tanto, la lealtad aparece como el componente con mayor contribución, de manera consistente con otros estudios (Delgado y Munuera, 2001; Arnett et al., 2003; Delgado, 2003), seguida de dos de las tres asociaciones de marca consideradas.

La calidad percibida presenta un efecto no significativo en ambas muestras. Una posible explicación a esta influencia no significativa puede encontrarse en las marcas incluidas en el estudio. Así el hecho de que todas las marcas seleccionadas gocen de una elevada calidad percibida tanto para los consumidores españoles como para los ingleses, puede haber originado que esta variable no tenga un papel predominante (Wang et al., 2006).

La notoriedad de marca muestra una influencia significativa sólo en España. Sin embargo, su efecto es negativo. A pesar de que la notoriedad es un indicador ampliamente utilizado en la literatura, algunos estudios han concluido que su uso para la valoración del valor de las marcas no es adecuado (Agarwal y Rao, 1996; Delgado y Munuera, 2001; Maio Mackay, 2001). Este hecho se debe a que la notoriedad puede llegar a adquirir un carácter secundario o irrelevante cuando todos los consumidores son conocedores de las marcas analizadas (Maio Mackay, 2001), siendo de este modo insuficiente para crear valor. El resultado obtenido podría venir explicado asimismo por las correlaciones existentes entre las dimensiones. A pesar de no haberse detectado problemas de colinealidad, las correlaciones existentes entre las variables independientes podrían haber influido sobre este parámetro.

Finalmente, la influencia de las asociaciones organizacionales difiere en función del país analizado. Así, en el Reino Unido las asociaciones organizacionales tienen un efecto significativo y positivo sobre el valor de marca, lo que sugiere que la medición del valor de marca en este contexto debería incluir este tipo de asociaciones. Al contrario, el resultado obtenido en España muestra que este tipo de asociaciones no contribuyen de forma significativa a la formación del valor de marca y que, por tanto, no parecen ser lo bastante importantes como para ser consideradas un componente del instrumento de medición propuesto.

En ambos casos el modelo presentó un ajuste aceptable. Asimismo, los valores de los coeficientes $\mathrm{R}^{2}$ mostraron un nivel suficiente de explicación de la varianza (España $\mathrm{R}^{2}=$ 0,714; Reino Unido $\mathrm{R}^{2}=0,622$ ).

Una vez realizado este análisis, con el objetivo de proporcionar una evidencia mayor en torno a la validez del instrumento de medición en ambos países, siguiendo las recomendaciones de Diamantopoulos y Winklhofer (2001), éste se vinculó a dos constructos con los que teóricamente se relaciona (validación nomológica). En concreto se seleccionaron las siguientes variables: la disposición del consumidor a pagar un precio primado y la intención de compra. Esta prueba, que permite valorar si la composición final del instrumento de medición es adecuada, arrojó unos resultados positivos. En este sentido, los coeficientes que relacionan el índice del valor de marca con las variables empleadas fueron significativos en ambos países (tabla 5), lo que confirma la validez externa. El ajuste del modelo también fue satisfactorio. 
Tabla 5

Resultados de la validación nomológica

\begin{tabular}{c|c|c|c|c}
\hline \multirow{2}{*}{ Relación } & \multicolumn{2}{|c|}{ España } & \multicolumn{2}{c}{ Reino Unido } \\
\cline { 2 - 5 } & Coef. (error) & $\mathrm{R}^{2}$ & Coef. (error) & $\mathrm{R}^{2}$ \\
\hline Índice del valor de marca $\rightarrow$ Precio primado & $0,686^{*}(8,174)$ & 0,471 & $0,792 *(6,870)$ & 0,627 \\
\hline Índice del valor de marca $\rightarrow$ Intención compra & $0,939 *(7,183)$ & 0,881 & $0,836 *(6,645)$ & 0,698 \\
\hline
\end{tabular}

Bondad de ajuste del modelo: España

$\mathrm{S}-\mathrm{B} \chi^{2}(\mathrm{df}, \mathrm{p})=422,79(123,0,00) ; \mathrm{RMSEA}=0,077$;

$\mathrm{NFI}=0,888 ; \mathrm{NNFI}=0,897 ; \mathrm{CFI}=0,917 ; \mathrm{IFI}=0,918$

Bondad de ajuste del modelo: Reino Unido

$\mathrm{S}-\mathrm{B} \chi^{2}(\mathrm{df}, \mathrm{p})=160,16(92,0,00) ; \mathrm{RMSEA}=0,043$;

$\mathrm{NFI}=0,969 \mathrm{NNFI}=0,982 ; \mathrm{CFI}=0,986 ; \mathrm{IFI}=0,987$

Nota: $* \mathrm{p}<0,05$

\subsection{Análisis de la invarianza en la medición}

Una vez analizada la validez del instrumento de medición propuesto de manera individual para cada país, a continuación, se procedió al análisis de la invarianza en la medición. Para ello, se siguieron los pasos propuestos por Diamantopoulos y Papadopoulos (2010).

\section{A. Análisis preliminares: valoración de la invarianza métrica de los indicadores reflectivos}

El primer paso consistió en la valoración de la invarianza métrica de los indicadores reflectivos utilizados, esto es, tanto de los indicadores de cada una de las dimensiones del valor de marca, como de los indicadores reflectivos que resumen la esencia del concepto y que son necesarios para la estimación del modelo MIMIC. Este paso es imprescindible, ya que la ausencia de invarianza métrica en estos parámetros afectaría a la fiabilidad de los resultados posteriores. En este sentido, la ausencia de invariabilidad impediría conocer si las diferencias observadas en las estimaciones de los pesos o en los errores son reales o consecuencia de la variabilidad de los indicadores reflectivos.

Para este análisis preliminar se tomaron como punto de partida las escalas validadas de manera individual en cada muestra en el epígrafe anterior. Como puede observarse en la tabla 6, las restricciones de igualdad para todas las cargas factoriales pueden ser sostenidas, al no existir un incremento significativo en el valor de la $\mathrm{S}-\mathrm{B} \chi^{2}$ en relación con el modelo anterior. De manera similar, el resto de índices siguen mostrando unos niveles de ajuste aceptables. Por tanto, puede concluirse que el criterio de invarianza métrica se cumple. 
Tabla 6

Análisis de la invarianza en la medición: indicadores reflectivos

\begin{tabular}{l|c|c|c|c|c|c|c|c}
\hline \multicolumn{1}{c|}{ Etapas } & $\begin{array}{c}\mathrm{S}-\mathrm{B} \chi^{2} \\
(\mathrm{~g} .1 ., \mathrm{p})\end{array}$ & $\mathrm{CFI}$ & $\mathrm{IFI}$ & $\mathrm{NFI}$ & $\mathrm{NNFI}$ & RMSEA & $\Delta \mathrm{S}-\mathrm{B} \chi^{26}$ & $\Delta \mathrm{g} .1$. \\
\hline $\begin{array}{l}\text { Invarianza } \\
\text { configural }\end{array}$ & $\begin{array}{c}1121,37 \\
(462,0,000)\end{array}$ & 0,943 & 0,944 & 0,908 & 0,932 & 0,059 & - & - \\
\hline $\begin{array}{l}\text { Invarianza } \\
\text { métrica }\end{array}$ & $\begin{array}{c}1139,67 \\
(479,0,000)\end{array}$ & 0,943 & 0,944 & 0,906 & 0,935 & 0,058 & $17,41^{\text {n.s. }}$ & 17 \\
\hline
\end{tabular}

Nota: n.s.: no significativo.

\section{B. Análisis de la invarianza en la estructura}

Este primer nivel de invarianza, como ya se ha adelantado, supone obtener un modelo MIMIC base (M1), en el cual tan sólo se imponen restricciones de igualdad en las cargas factoriales de los indicadores reflectivos. La estimación simultánea de los parámetros para cada grupo arrojó los resultados recogidos en la tabla 7. Como puede observarse, los índices de bondad de ajuste de este modelo revelan un adecuado ajuste.

${ }^{6}$ Dado que los análisis están basados en el método de estimación de máxima verosimilitud robusto, la comparación de los modelos anidados basada en la diferencia del valor de la $\chi^{2}$ de Satorra-Bentler requiere de una corrección previa, ya que la variación en este valor no se distribuye como una $\chi^{2}$. El calculo para realizar esta corrección es el siguiente:

$$
\Delta S-B \chi^{2}=\frac{M L \chi_{1}^{2}-M L \chi_{0}^{2}}{\left(g_{1} \frac{M L \chi_{1}^{2}}{S-B \chi_{1}^{2}}-g_{0} \frac{M L \chi_{0}^{2}}{S-B \chi_{0}^{2}}\right) /\left(g_{1}-g_{0}\right)} \text { dónde } g_{0} \text { y } g_{1} \text { son los grados de }
$$

libertad del modelo base y del modelo restringido respectivamente; $M L \chi_{0}^{2}$ y $M L \chi_{1}^{2}$ son los valores de la chicuadrado del método de máxima verosimilitud del modelo base y del modelo restringido respectivamente; y $S$ - $B$

$\chi_{0}^{2}$ y $S-B \chi_{1}^{2}$ son los valores de la chi-cuadrado de Satorra-Bentler del modelo base y del modelo restringido respectivamente (Byrne, 2006). 
Tabla 7

Análisis de la invarianza en la medición: constructo formativo

\begin{tabular}{l|c|c|c|c|c|c|c|c|c}
\hline \multicolumn{1}{c|}{ Etapas } & Modelo & $\begin{array}{c}\text { S-B } \chi^{2} \\
(\mathrm{~g} .1 ., \mathrm{p})\end{array}$ & $\mathrm{CFI}$ & $\mathrm{IFI}$ & $\mathrm{NFI}$ & $\mathrm{NNFI}$ & $\mathrm{RMSEA}$ & $\Delta \mathrm{S}-\mathrm{B} \chi^{27}$ & $\Delta \mathrm{g} .1$. \\
\hline $\begin{array}{l}\text { Invarianza en } \\
\text { la estructura }\end{array}$ & $\mathrm{M} 1$ & $\begin{array}{c}1022,93 \\
(434,0,000)\end{array}$ & 0,945 & 0,946 & 0,909 & 0,936 & 0,058 & - & - \\
\hline $\begin{array}{l}\text { Invarianza en } \\
\text { los pesos }\end{array}$ & $\mathrm{M} 2$ & $\begin{array}{c}1042,37 \\
(440,0,000)\end{array}$ & 0,944 & 0,944 & 0,907 & 0,936 & 0,058 & $19,55^{*}$ & 6 \\
\hline $\begin{array}{l}\text { Invarianza } \\
\text { parcial en los } \\
\text { pesos }\end{array}$ & $\mathrm{M} 3$ & $\begin{array}{c}1024,74 \\
(437,0,000)\end{array}$ & 0,945 & 0,946 & 0,909 & 0,937 & 0,057 & $1,86^{\text {n.s. }}$ & 3 \\
\hline $\begin{array}{l}\text { Invarianza } \\
\text { parcial en los } \\
\text { residuos }\end{array}$ & $\mathrm{M} 4$ & $\begin{array}{c}1024,75 \\
(438,0,000)\end{array}$ & 0,945 & 0,946 & 0,909 & 0,937 & 0,057 & $1,88^{\text {n.s. }}$ & 4 \\
\hline
\end{tabular}

Nota: n.s.: no significativo; $* \mathrm{p}<0,05$.

\section{Análisis de la invarianza en los pesos}

Este nivel de invarianza constituye un test más estricto cuyo examen exige imponer la restricción de igualdad en los pesos, con el objetivo de determinar si éstos son equivalentes en los dos grupos. Para probar su existencia, se compara el ajuste de este nuevo modelo (M2) con los índices del modelo anterior (M1), según los criterios anteriormente comentados. En caso de encontrar diferencias significativas, a través del test multiplicador de Lagrange se debe analizar qué pesos carecen de equivalencia, para de este modo comprobar la existencia de invarianza parcial.

Los resultados de la tabla 7 muestran un incremento significativo en el valor de la S-B $\chi^{2}$ en relación con el modelo anterior, a pesar de que el resto de índices siguen mostrando unos niveles de ajuste aceptables. Un examen detallado del test multiplicador de Lagrange reveló que este aumento significativo era debido a las restricciones en los pesos relativos a la notoriedad y la lealtad. La relajación secuencial de estas dos restricciones dio lugar a un modelo parcialmente invariante que produjo un aumento de la chi-cuadrado no significativo $\left(\Delta \mathrm{S}-\mathrm{B} \chi^{2}=7,85 ; \Delta \mathrm{g} .1 .=4 ; \mathrm{p}>0,05\right)$. El test multiplicador de Lagrange sugería, no obstante, la eliminación de la restricción en el peso relativa a las asociaciones organizacionales. Por ello, un examen más estricto de la invariabilidad llevó al modelo M3.

\section{Análisis de la invarianza en los residuos}

Siguiendo las recomendaciones de Diamantopoulos y Papadopoulos (2010), a continuación se procedió a contrastar el último nivel de invarianza: la invarianza en los residuos.

\footnotetext{
${ }^{7}$ Ver nota anterior.
} 
Para ello, se añadió al modelo M3 la restricción adicional de igualdad en este parámetro entre ambas muestras y, de manera análoga al procedimiento realizado anteriormente, se comparó el ajuste de este modelo con el modelo base (M1).

Como puede observarse en la tabla 7 , no se produjo un incremento significativo en el valor de la $\mathrm{S}-\mathrm{B} \chi^{2}$. Sobre la base de estos resultados, puede concluirse que los errores son equivalentes en las dos muestras.

La tabla 8 muestras las estimaciones de los parámetros en el modelo final (M4). De los seis componentes, tres resultaron invariantes. Por el contrario, la lealtad contribuye positivamente a la formación del valor de marca en ambos países, aunque su importancia en España es significativamente mayor. Asimismo, los consumidores ingleses parecen dar una mayor importancia a las asociaciones de carácter organizacional, mientras que la notoriedad tiene una influcencia significativa, aunque negativa, solamente en el caso español.

Tabla 8

Parámetros no estandarizados y errores

\begin{tabular}{l|c}
\hline \multicolumn{1}{c|}{ Componente } & Parámetro no estandarizado (error) \\
\hline Notoriedad & España: $-0,349 *(-3,427) ;$ Reino Unido: $-0,113(-1,420)$ \\
\hline Calidad percibida & $0,090(1,430)$ \\
\hline Lealtad & España: $0,608^{*}(12,497) ;$ Reino Unido: $0,384 *(7,420)$ \\
\hline Valor percibido (asoc.) & $0,340 *(3,658)$ \\
\hline Personalidad de la marca (asoc.) & $0,227 *(3,415)$ \\
\hline Asoc. Organizacionales (asoc.) & España: $-0,024(-0,332) ;$ Reino Unido: $0,201 *(2,652)$ \\
\hline
\end{tabular}

Nota: $* \mathrm{p}<0,05$.

Finalizado el análisis de invarianza en la medición, las diferencias identificadas sugieren, por tanto, que el constructo de valor de marca se caracteriza por presentar variaciones en su composición en los países analizados. La ausencia de un acuerdo en torno al número de parámetros que deberían ser invariantes (Diamantopoulos y Papadopoulos, 2010), nos impide, no obstante, realizar una valoración más precisa del grado e importancia de estas diferencias.

\section{CONCLUSIONES}

Este estudio ha conceptualizado el valor de marca como un constructo de segundo orden con componentes formativos y ha validado empíricamente dicha conceptualización en un escenario cross-nacional, concretamente en dos países europeos: España y el Reino Unido. Con este fin, se ha realizado un análisis de la invarianza en la medición, además de un análisis individual para cada país. Para ello se ha llevado a cabo un estudio de mercado en ambos países, obteniéndose 411 cuestionarios válidos en cada uno de ellos. 
A través de esta conceptualización del valor de marca usando un enfoque formativo, este trabajo ha superado limitaciones de estudios previos, en los que se sigue el paradigma de medición dominante en marketing (enfoque reflectivo). Asimismo, constituye un avance sobre aquellas investigaciones que, habiendo utilizado un enfoque formativo, han conceptualizado el valor de marca como un constructo de primer orden (Delgado y $\mathrm{Mu}$ nuera, 2001; Arnett et al., 2003; Delgado, 2003). Por último, se ha realizado un análisis de invarianza en la medición siguiendo el reciente y novedoso procedimiento propuesto por Diamantopoulos y Papadopoulos (2010).

A partir de una exhaustiva revisión de la literatura, el valor de marca se ha conceptualizado como un constructo multidimensional, compuesto por los siguientes componentes: notoriedad de la marca, calidad percibida, asociaciones del nombre de marca y lealtad a la marca. Tras justificar la naturaleza formativa de dichas dimensiones, se ha procedido a la validación del instrumento de medición propuesto de manera individual en cada país. Por último, se ha realizado un análisis de la invarianza en la medición.

Los resultados obtenidos nos permiten extraer las siguientes conclusiones. En ambos países, la validación del instrumento de medición propuesto presenta semejanzas. Así, tanto la lealtad a la marca como dos de las asociaciones de marca consideradas (valor percibido y personalidad de marca) contribuyen de manera significativa al mismo.

De igual modo, la calidad percibida parece no ser un indicador relevante en ninguno de los mercados. Por su parte, la notoriedad, contrariamente a lo esperado, tiene una influencia negativa en el caso español.

Como se ha apuntado anteriormente, el hecho de que todas las marcas tuvieran una alta notoriedad entre los consumidores, y que éstos las consideraran de calidad medio-alta, junto con las correlaciones existentes entre las variables independientes, ha podido condicionar estos resultados. Por consiguiente, en futuras investigaciones sería especialmente interesante analizar la contribución de estas dos variables y, de este modo, confirmar la idoneidad o no de incluirlas en la medición del valor de marca.

Finalmente, de entre los componentes analizados, las asociaciones organizacionales presentan una contribución diferente en función del país en el que se valide el instrumento de medición propuesto.

El análisis de la invarianza en la medición, por el cual se ha comprobado la existencia de los diferentes niveles de invarianza propuestos en la literatura, ha corroborado las diferencias inicialmente identificadas en los análisis individuales. En este sentido, se ha podido comprobar la existencia de un nivel de invariabilidad parcial. Por tanto, los resultados obtenidos ponen de manifiesto que el constructo en sí mismo presenta diferencias entre los países analizados.

Las conclusiones obtenidas nos permiten plantear un conjunto de implicaciones de interés tanto para académicos como profesionales. En primer lugar, el trabajo presentado contribuye a la literatura existente en torno a la conceptualización del valor de marca. Además, extiende las aplicaciones de indicadores formativos realizadas hasta la fecha, caracterizadas fundamentalmente por la utilización de constructos unidimensionales, al proponer un modelo de segundo orden y examinar su invariabilidad en diferentes países.

A nivel de gestión, de acuerdo con los resultados obtenidos en el estudio, los gerentes deberían ser conscientes de que el valor de marca puede venir condicionado por diferentes componentes. En el presente estudio, estas diferencias se han identificado al nivel del país. 
En este sentido, el análisis del instrumento de medición muestra un elevado grado de coincidencia entre los dos países analizados, España y el Reino Unido. Sin embargo, también presenta algunas diferencias (por ejemplo, en la notoriedad, la lealtad y las asociaciones organizacionales).

El instrumento de medición del valor de marca, una vez adaptado a las peculiaridades de cada país objeto de análisis, puede resultar de gran utilidad para las empresas. Así, las organizaciones pueden utilizar esta herramienta para obtener una adecuada comprensión de los componentes que mayor contribución tienen en la creación del valor de marca. De este modo, esta información permitirá realizar una distribución más eficiente de los recursos disponibles. Asimismo, el instrumento de medición puede ser utilizado para evaluar la efectividad de las decisiones y estrategias puestas en marcha o realizar análisis comparativos con las marcas competidoras, entre otros fines.

La investigación realizada no está libre de limitaciones. Cabe destacar en primer lugar la conceptualización del constructo objeto de estudio. En la investigación desarrollada se analizan las dimensiones que mayor aceptación, tanto desde un punto de vista teórico como práctico, han tenido en la literatura. En futuras investigaciones sería interesante examinar cómo la incorporación de otros componentes podría mejorar el instrumento de medición propuesto. Asimismo, desde una perspectiva metodológica, en esta investigación se han analizado unas categorías de producto y marcas concretas, por lo que habría que considerar otros tipos de productos, servicios y marcas para extrapolar los resultados. De la misma forma, los resultados obtenidos son válidos en los dos países considerados: España y el Reino Unido. Futuras líneas de investigación deberían incorporar nuevos países. Por último, y dentro de las limitaciones propias de las medidas formativas, destacaría el efecto que la elección de los indicadores reflectivos incluidos en la estimación del modelo MIMIC pueda ejercer en los resultados obtenidos. 
Apéndice

Escalas empleadas en los cuestionarios

NOTORIEDAD (Yoo et al., 2000; Netemeyer et al., 2004)

NOT1. He oído hablar de la marca $X$

NOT2. Cuando pienso en $\mathrm{CP}, \mathrm{X}$ es una de las marcas que me viene a la mente

NOT3. La marca $X$ me resulta familiar

NOT4. Conozco la marca $\mathrm{X}$

NOT5. Puedo reconocer la marca $X$ frente a otras marcas competidoras de $\mathrm{CP}$

CALIDAD PERCIBIDA (Pappu et al., 2005, 2006)

CP1. X ofrece productos de muy buena calidad

$\mathrm{CP} 2$. Los productos de $\mathrm{X}$ tienen una calidad consistente

CP3. Los productos de X son de confianza

$\mathrm{CP} 4$. Los productos de $\mathrm{X}$ tienen unas características excelentes

LEALTAD (Yoo et al., 2000)

LEA1. Me considero un consumidor leal a la marca $\mathrm{X}$

LEA2. Si comprara CP, X sería mi primera opción de compra

LEA3. No compraría otras marcas de CP si X estuviera disponible en el establecimiento

ASOCIACIONES DE LA MARCA

VALOR PERCIBIDO (Lassar et al., 1995; Aaker, 1996; Netemeyer et al., 2004)

ASO1. La marca X tiene una buena relación calidad-precio

ASO2. Dentro del mercado de categoría de producto, considero que X es una buena compra

ASO3. La marca $X$ aporta un elevado valor con relación al precio que hay que pagar por ella

PERSONALIDAD de la MARCA (Aaker, 1996)

ASO4. La marca $\mathrm{X}$ tiene personalidad

ASO5. La marca $X$ es interesante

ASO6. Tengo una clara imagen del tipo de personas que consumen la marca $\mathrm{X}$

ASOCIACIONES ORGANIZACIONALES (Aaker, 1996; Pappu et al., 2005, 2006)

ASO7. Confío en la empresa que fabrica la marca $X$

ASO8. Me gusta la empresa que fabrica la marca $X$

ASO9. La empresa que fabrica la marca $X$ tiene credibilidad

VALOR DE MARCA (medida reflectiva) (Yoo et al., 2000)

VM1. Tiene sentido comprar la marca $\mathrm{X}$ en lugar de otras marcas de CP, aunque sean iguales

VM2. Aun habiendo otras marcas de CP con las mismas características, yo preferiría comprar la marca X

VM3. Aunque hubiera otras marcas de $\mathrm{cp}$ tan buenas como $\mathrm{X}$, yo preferiría comprar $\mathrm{X}$

VM4. Aunque $\mathrm{X}$ no fuera diferente a otras marcas de CP, es más inteligente comprar $\mathrm{X}$

DISPOSICIÓN A PAGAR UN PRECIO PRIMADO (Netemeyer et al., 2004)

PRE1. El precio de $X$ tendría que subir bastante para que no considerara comprarla

PRE2. Estoy dispuesto a pagar un precio mayor por la marca $\mathrm{X}$ que por otras marcas de $\mathrm{CP}$

PRE3. Estoy dispuesto a pagar mucho más por la marca $X$ que por otras marcas de $\mathrm{CP}$

INTENCIÓN DE COMPRA (Erdem et al., 2006)

COM1. Compraría categoría de producto de la marca $\mathrm{X}$

COM2. Definitivamente, consideraría comprar la marca $\mathrm{X}$

COM3. Es muy probable que compre la marca $\mathrm{X}$

Nota: CP: Categoría de producto 


\section{REFERENCIAS BIBLIOGRÁFICAS}

AAKER, D.A. (1991): Managing Brand Equity Capitalizing on the Value of Brand Name, New York: The Free Press.

AAKER, D.A. (1996): "Measuring Brand Equity Across Products and Markets", California Management Review, Vol. 38, No. 3, pp. 102-120.

AGARWAL, M.K. Y RAO, V.R. (1996): "An Empirical Comparison of Consumer-Based Measures of Brand Equity", Marketing Letters, Vol. 7, No. 3, pp. 237-247.

AILAWADI, K.L.; LEHMANN, D.R. Y NESLIN, S.A. (2003): "Revenue Premium as an Outcome Measure of Brand Equity", Journal of Marketing, Vol. 67, pp. 1-17.

ARNETT, D.B.; LAVERIE, D.A. Y MEIERS, A. (2003): "Developing Parsimonious Retailer Equity Indexes Using Partial Least Squares Analysis: A Method and Applications", Journal of Retailing, Vol. 79, pp. 161-170.

ARNETT, D.B.; WILCOX, J.B. Y HOWELL, R.D. (2009): "Measuring Brand Equity: Issues for Marketing Academicians and Practitioners", The Thought Leaders International Conference on Brand Management, Atenas.

ASHILL, N.J. Y SINHA, A. (2004): "An Exploratory Study into the Impact of Components of Brand Equity and Country of Origin Effects on Purchase Intention", Journal or Asia-Pacific Business, Vol. 5, No. 3, pp. 27-43.

ATILGAN, E.; AKSOY, S. Y AKINCI, S. (2005): "Determinants of the Brand Equity: A Verification Approach in the Beverage Industry in Turkey", Marketing Intelligence \& Planning, Vol. 23, No. 2/3, pp. 237-248.

BAUER, H.H.; SAUER, N.E. Y SCHMITT, P. (2005): "Customer-based Brand Equity in the Team Sport Industry: Operationalization and Impact on the Economic Success of Sport Teams", European Journal of Marketing, Vol. 39, No. 5/6, pp. 496-513.

BELSLEY, D.A. (1991): Conditioning Diagnostics: Collinearity and Weak Data in Regression. Wiley \& Sons, New York.

BLACKSTON, M. (1990): "Price Trade-offs as a Measure of Brand Value", Journal of Advertising Research, Vol. 30, pp. 3-6.

BOLLEN, K. Y LENNOX, R. (1991): “Conventional Wisdom on Measurement: A Structural Equation Perspective», Psychological Bulletin, Vol. 110, No. 2, pp. 305-314.

BRAVO, R.; FRAJ, E. Y MARTÍNEZ, E. (2007): "Family as a Source of Consumer-based Brand Equity”, Journal of Product and Brand Management, Vol. 16, No. 3, pp. 188-199.

CHEN, K.J. (2001): "Using Free Association to Examine the Relationship between the Characteristics of Brand Associations and Brand Equity", Journal of Product and Brand Management, Vol. 10, No. 7, pp. 439-451.

CHRISTODOULIDES, G. Y DE CHERNATONY, L. (2004): "Dimensionalising On- and Offline Brands' Composite Equity", Journal of Product and Brand Management, Vol. 13, No. 3, pp. 168-179.

COBB-WALGREN, C.; RUBLE, C.A. Y DONTHU, N. (1995): "Brand Equity, Brand Preference and Purchase Intent", Journal of Advertising, Vol. 24, No. 3, pp. 25-40.

CRAIG, C.S. Y DOUGLAS, S.P. (2005): International Marketing Research, $3^{\text {a }}$ Ed., London, UK: John Wiley \& Sons.

DE CHERNATONY, L.; HARRIS, F. Y CHRISTODOULIDES, G. (2004): "Developing a Brand Performance Measure for Financial Services Brands", The Service Industries Journal, Vol. 24, No. 2 (marzo), pp. 15-33.

DEAN, D.H. (2004): "Evaluating Potential Brand Associations through Conjoint Analysis and Market Simulation", Journal of Product and Brand Management, Vol. 13, No. 7, pp. 506-513.

DEL RÍO, A.B.; VÁZQUEZ, R. E IGLESIAS, V. (2001): "The Effects of Brand Associations on Consumer Response", Journal of Consumer Marketing, Vol. 18, No. 5, pp. 410-425. 
DEL RÍO, A.B.; VÁZQUEZ, R. E IGLESIAS, V. (2002): "El Valor de Marca: Perspectivas de Análisis y Criterios de Estimación”, Cuadernos de Gestión, Vol. 1, No. 2, pp. 87-102.

DELGADO, E. (2003): "Controversia Conceptual sobre el Capital de Marca: Propuesta de un Marco Teórico de Análisis", Revista Europea de Dirección y Economía de la Empresa, Vol. 12, No. 3, pp. 7-24.

DELGADO, E. Y MUNUERA, J.L. (2001): "Medición del Capital de Marca con Indicadores Formativos", Investigación y Marketing, Vol. 75, pp. 16-20.

DIAMANTOPOULOS, A. (2006): "The Error Term in Formative Measurement Models: Interpretation and Modelling Implications", Journal of Modelling Management, Vol. 1, pp. 7-17.

DIAMANTOPOULOS, A. Y PAPADOPOULOS (2010): "Assesing the Cross-national Invariance of Formative Measures: Guidelines for International Business Researchers", Journal of International Business Studies, Vol. 41, pp. 360-370.

DIAMANTOPOULOS, A.; RIEFLER, P. Y ROTH, K.P. (2008): "Advancing Formative Measurement Models", Journal of Business Research, Vol. 61, No. 12, pp. 1203-1218.

DIAMANTOPOULOS, A. Y WINKLHOFER, H.M. (2001): "Index Construction with Formative Indicators: An Alternative to Scale Development", Journal of Marketing Research, Vol. 38, mayo, pp. 269-277.

EDWARDS, J.R. (2001): "Multidimensional Constructs in Organizational Behavior Research: An Integrative Analytical Framework». Organizational Research Methods, vol. 4, núm. 2, págs. 144-192.

ERDEM, T.; SWAIT, J. Y VALENZUELA, A. (2006): "Brands as Signals: A Cross-country Validation Study", Journal of Marketing, Vol. 70, enero, pp. 34-49.

FARQUHAR, P.H. (1989): "Managing Brand Equity", Marketing Research, pp. 24-33.

FERNÁNDEZ, M. Y GONZÁLEZ, M. (2006): "Brand Equity in the European Fruit and Vegetable Sector: A Transaction Cost Approach", International Journal of Research in Marketing, Vol. 23, pp. 31-44.

FORNELL, C. (1982): A Second Generation of Multivariate Analysis. Praeger, New York.

GROVER, R. Y SRINIVASAN, V. (1992): "Evaluationg the Multiple Effects of Retail Promotions on Brand Loyal and Brand Switching Segments", Journal of Marketing Research, Vol. 29, febrero, pp. 76-89.

HAIR, J.F.; BLACK, B.; BABIN, B.; ANDERSON, R.E. Y TATHAM, R.L. (2006): Multivariate Data Analysis, $6^{\text {a }}$ Ed., Upper Saddle River, Prentice Hall, N.J.

HOFSTEDE, G. (2001): Culture's Consequences, Comparing Values, Behaviors, Institutions, and Organizations across Nations, Thousand Oaks CA: Sage Publications.

Hsien, M.H. (2002): "Identifying Brand Image Dimensionality and Measuring the Degree of Brand Globalization: A Cross-National Study", Journal of International Marketing, Vol. 10, No. 2, pp. 46-67.

HsieH, M.H. (2004): "Measuring Global Brand Equity Using Cross-National Survey Data". Journal of International Marketing, Vol. 12, No. 2, pp. 28-57.

HULT, G.T.M.; KETCHEN, D.J.; GRIFFITH, D.A.; FINNEGAN, C.A.; GONZALEZ-PADRON, T.; HARMANCIOGLU, N.; HUANG, Y.; TALAY, M.B. Y CAVUSGIL, S.T. (2008): "Data Equivalence in Cross-Cultural International Business Research: Assessment and Guidelines", Journal of International Business Studies, Vol. 39, No. 6, pp. 1027-1044.

INTERBRAND. BEST GLOBAL BRANDS 2006. A Ranking by Brand Value. Disponible en: http://www.interbrand.com/best brands 2006.asp.

JARVIS, C.B.; MACKENZIE, S.B. Y PODSAKOFF, P.M. (2003): “A Critical Review of Construct Indicators and Measurement Model Misspecification in Marketing and Consumer Research", Journal of Consumer Research, Vol. 30, pp. 199-218.

JUNG, J. Y SUNG, E-Y. (2008): "Consumer-based Brand Equity: Comparisons among Americans and South Koreans in the USA and South Koreans in Korea", Journal of Fashion Marketing and Management, Vol. 12, No. 1, pp. 24-35. 
KELLER, K.L. (1993): "Conceptualizing, Measuring, and Managing Customer-Based Brand Equity”, Journal of Marketing, Vol. 57, pp. 1-22.

KELLER, K.L. (2007): Strategic Brand Management: Building, Measuring, and Managing Brand Equity. $3^{\text {a }}$ Ed. New York: Prentice Hall.

KELLER, K.L. Y LEHMANN, D.R. (2006): "Brands and Branding: Research Findings and Future Priorities", Marketing Science, Vol. 25, No. 6, pp. 740-759.

KIM H. Y KIM, W.G. (2005): "The Relationship between Brand Equity and Firms' Performance in Luxury Hotels and Chain Restaurants", Tourism Management, Vol. 26, pp. 549-560.

KIM, K.H.; KIM, K.S.; KIM, D.Y.; KIM, J.H. Y KANG, S.H. (2008): "Brand Equity in Hospital Marketing", Journal of Business Research, Vol. 61, pp. 75-82.

KLINE, R.B. (2005): Principles and Practice of Structural Equation Modeling, $2^{\mathrm{a}}$ Ed., The Guilford Press, New York.

KOÇAK, A.; ABIMBOLA, T. Y ÖZER, A. (2007): "Consumer Brand Equity in a Cross-cultural Replication: An Evaluation of a Scale", Journal of Marketing Management, Vol. 23, No. 1/2, pp. 157-173.

KONECNIK, M. Y GARTNER, W.C. (2007): “Customer-Based Brand Equity for a Destination", Annals of Tourism Research, Vol. 34, No. 2, pp. 400-421.

KRISHNAN, H.S. (1996): "Characteristics of memory associations: A consumer-based brand equity perspective", International Journal of Research in Marketing, Vol. 13, pp. 389-405.

KUMAR, V. (2000): International Marketing Research, Prentice Hall, Upper saddle River, NJ.

LASSAR, W.; MITTAL, B. Y SHARMA, A. (1995): "Measuring Customer-based Brand Equity", Journal of Consumer Marketing, Vol. 12, No. 4, pp. 11-19.

LEE, J-S. Y BACK, K-J. (2008): “Attendee-based Brand Equity”, Tourism Management, Vol. 29, pp. 331-344.

LEONE, R.P.; RAO, V.R.; KELLER, K.L.; LUO, A.M.; MCALISTER, L. Y SRIVASTAVA, R. (2006): "Linking Brand Equity to Customer Equity", Journal of Service Research, Vol. 9, No. 2, pp. 125-138.

LOW, G.S. Y LAMB, CH.W. (2000): “The Measurement and Dimensionality of Brand Association”, Journal of Product and Brand Management, Vol. 9, No. 6, pp. 350-368.

MACKENZIE, S.B.; PODSAKOFF, P.M. Y JARVIS, C.B. (2005): “The Problem of Measurement Model Misspecification in Behavioral and Organizational Research and some Recommended Solutions", Journal of Applied Psychology, Vol. 90, No. 4, pp. 710-730.

MAIO MACKAY, M. (2001): "Evaluation of Brand Equity Measures: Further Empirical Results", Journal of Product and Brand Management, Vol. 10, No. 1, pp. 38-51.

MALHOTRA, N.K.; PETERSON, M. Y KLEISER, S.B. (1999): "Marketing Research: A State-ofthe-Art Reivew and Diretions for the Twenty-First Century", Journal of the Academy of Marketing Science, Vol. 27, No. 2, pp. 160-183.

MARTIN, G.S. Y BROWN, T.J. (1990): "In Search of Brand Equity: The Conceptualization and Measurement of the Brand Impression Construct", en M.L. Childers et al. (eds.): Marketing Theory and Applications, Vol. 2 (pp. 431-438), Chicago: American Marketing Association.

MOHD, N.; NASSER, M. Y MOHAMAD, O. (2007): "Does Image of Country-of-origin Matter to Brand Equity?", Journal of Product and Brand Management, Vol. 16, No. 1, pp. 38-48.

NETEMEYER, R.G.; KRISHNAN, B.; PULLIG, C.; WANG, G.; YAGCI, M.; DEAN, D.; RICKS, J. Y WIRTH, F. (2004): "Developing and Validating Measures of Facets of Customer-based Brand Equity", Journal of Business Research, Vol. 57, pp. 209-224.

PAPPU, R.; QUESTER, P.G. Y COOKSEY, R.W. (2005): “Consumer-based Brand Equity: Improving the Measurement. Empirical Evidence", Journal of Product and Brand Management, Vol. 14, No. 3, pp. 143-154.

PAPPU, R.; QUESTER, P.G. Y COOKSEY, R.W. (2006): "Consumer-based Brand Equity and Country-of-origin Relationships”, European Journal of Marketing, Vol. 40, No. 5/6, pp. 696-717. 
PAPPU, R.; QUESTER, P.G. Y COOKSEY, R.W. (2007): "Country Image and Consumer-based Brand Equity: Relationships and Implications for International Marketing", Journal of International Business Studies, Vol. 38, pp. 726-745.

PARK, C.W.; MACINNIS, D.J.; DREZE, X. Y LEE, J. (2009): "Measuring Brand Equity: The Marketing Surplus and Efficiency (MARKSURE) Based Brand Equity Measure", en Loken, B., Ahluwalia, R. y Houston, M. (Ed.), Contemporary Branding Issues: A Research Perspective, Taylor and Francis Group Publishing.

PODSAKOFF, N.; SHEN, W. Y PODSAKOFF, P.M. (2006): “The Role of Formative Measurement Models in Strategic Management Research: Review, Critique, and Implications for Future Research", en Ketchen, D. y Bergh, D. (Ed.): Research Methodology in Strategic Management, Vol. 3, Elsevier, Oxford, England.

ROSSITER, J.R. Y PERCY, L.P. (1987): Advertising and Promotion Management, McGraw-Hill: New York.

SEETHARAMAN, A.; NADZIR, A.A.B.M. Y GUNALAN, S. (2001): "A Conceptual Study on Brand Valuation", Journal of Product and Brand Management, Vol. 10, No. 4, pp. 243-256.

STEENKAMP, J-B.E.M. Y BAUMGARTNER, H. (1998): "Assessing Measurement Invariance in Cross-National Consumer Research". Journal of Consumer Research, Vol. 25 (junio), pp. 78-90.

STEENKAMP, J-B.E.M. Y VAN TRIJP, H.C. (1991): "The Use of LISREL in the Validating Marketing Constructs", International Journal of Research in Marketing, Vol. 8, pp. 283-299.

SUPPHELLEN, M. (2000): "Understanding Core Brand Equity: Guidelines for In-depth Elicitation of Brand Associations", International Journal of Market Research, Vol. 42, No. 3, pp. 319-338.

SWAIT, J.; ERDEM, T.; LOUVIERE, J. Y DUBELAAR, C. (1993): "The Equalization Price: A Measure of Consumer-perceived Brand Equity", International Journal of Research in Marketing, Vol. 10, pp. 23-45.

VANDENBERG, R.J. Y LANCE, C.E. (2000): "A Review and Synthesis of the Measurement Invariance Literature: Suggestions, Practices, and Recommendations for Organizational Research", Organizational Research Methods, Vol. 3, No. 1, pp. 4-69.

VÁZQUEZ, R.; DEL RÍO, A.B. E IGLESIAS, V. (2002): “Consumer-Based Brand Equity: Development and Validation of a Measurement Instrument", Journal of Marketing Management, Vol. 39, No. 3/4, pp. 351-364.

VILLAREJO, A.F. (2001): La Medición del Valor de Marca en el Ámbito de la Gestión de Marketing, CEADE.

WANG, Y.; KANDAMPULLY, J.A.; LO, H. Y SHI, G. (2006): “The Roles of Brand Equity and Corporate Reputation in CRM: A Chinese Study", Corporate Reputation Review, Vol. 9, No. 3, pp. 179-197.

WASHBURN, J.H. Y PLANK, R.E. (2002): "Measuring Brand Equity: An Evaluation of a Consumer-based Brand Equity Scale", Journal of Marketing Theory and Practice, Vol. 10, No. 1, pp. 46-62.

WINTERS, L.C. (1991): "Brand Equity Measures: Some Recent Advances”, Marketing Research, pp. 70-73.

YOO, B. Y DONTHU, N. (2001): "Developing and Validating a Multidimensional Consumer-based Brand Equity Scale", Journal of Business Research, Vol. 52, pp. 1-14.

YOO, B. Y DONTHU, N. (2002): "Testing Cross-Cultural Invariance of Brand Equity Creation Process", Journal of Product and Brand Management, Vol. 11, No. 6, pp. 380-398.

YOO, B.; DONTHU, N. Y LEE, S. (2000): "An Examination of Selected Marketing Mix Elements and Brand Equity", Academy of Marketing Science, Vol. 28, No. 2, pp. 195-211.

ZEITHAML, V.A. (1988): "Consumer Perceptions of Price, Quality and Value: A Means-end Model and Synthesis of Evidence", Journal of Marketing, Vol. 52, julio, pp. 2-22. 